\title{
Endocuff Vision improves adenoma detection rate in a large screening-related cohort
}

\section{다 (우우}

\section{Authors}

Nauzer Forbes ${ }^{1,2}$, Robert J. Hilsden ${ }^{1,2}$, Yibing Ruan ${ }^{3,4}$, Abbey E. Poirier ${ }^{3,4}$, Dylan E. O’Sullivan ${ }^{3}$, Kyla M. Craig ${ }^{5}$, Diana Kerrison $^{5}$, Darren R. Brenner ${ }^{2,3,4}$, Steven J. Heitman ${ }^{1,2}$

\section{Institutions}

1 Department of Medicine, Cumming School of Medicine, University of Calgary, Calgary, Alberta, Canada

2 Department of Community Health Sciences, Cumming School of Medicine, University of Calgary, Calgary, Alberta, Canada

3 Department of Cancer Epidemiology and Prevention Research, CancerControl Alberta, Alberta Health Services, Calgary, Alberta, Canada

4 Department of Oncology, Cumming School of Medicine, University of Calgary, Calgary, Alberta, Canada

5 Alberta Health Services (Calgary Zone), Calgary, Alberta, Canada

submitted 24.11.2020

accepted after revision $\quad 6.5 .2021$

Bibliography

Endosc Int Open 2021; 09: E1583-E1592

DOI 10.1055/a-1533-6183

ISSN 2364-3722

(C) 2021. The Author(s).

This is an open access article published by Thieme under the terms of the Creative Commons Attribution-NonDerivative-NonCommercial License, permitting copying and reproduction so long as the original work is given appropriate credit. Contents may not be used for commercial purposes, or adapted, remixed, transformed or built upon. (https://creativecommons.org/licenses/by-nc-nd/4.0/)

Georg Thieme Verlag KG, Rüdigerstraße 14,

70469 Stuttgart, Germany

Corresponding author

Steven J. Heitman MD, MSc, FRCPC, Associate Professor of Medicine, TRW Building, 3280 Hospital Drive NW, Calgary, AB T2N 4 Z6

Fax: +1-403-592-5090

steven.heitman@ucalgary.ca

\section{ABSTRACT}

Background and study aims Endocuff Vision (ECV) increases adenoma detection rate (ADR) in randomized clinical trials; however, observational effectiveness data are lacking. We evaluated the effectiveness, safety, and practical aspects of ECV use in a large screening-related realworld cohort.

Patients and methods In this observational study, patients undergoing screening-related colonoscopy from November 2018 to April 2019 comprised the baseline period, and those undergoing it from June to November 2019 comprised the ECV period, where ECV use was discretionary. The primary outcome was ADR, compared: 1) between ECV use and standard colonoscopy across both periods; and 2) between time periods. Secondary outcomes included indication-specific ADR, sessile serrated ADR (SSADR), cecal intubation rate (CIR), procedure times, patient comfort scores, and sedation use. Multilevel logistic regression was performed, yielding adjusted odds ratios (AOR) with 95 $\%$ confidence intervals (Cls).

Results In 15,814 colonoscopies across both time periods, ADR was $46.7 \%$ with standard colonoscopy and $54.6 \%$ when ECV was used $(P<0.001)$. Endoscopists used ECV in $77.6 \%$ of procedures in the ECV period, during which overall ADR rose to $53.2 \%$ compared to $46.3 \%$ in the baseline period $(P<0.001)$. ECV use was significantly associated with higher ADR (AOR 1.24, $95 \% \mathrm{Cl} 1.10$ to 1.40 ) after adjusting for relevant covariates including time period. ECV use did not result in lower CIR, longer procedure time, increased sedation use, or poorer comfort scores.

Conclusions ECV use is associated with improved ADR without negatively impacting other key procedure and patient-related factors. Future studies should evaluate the cost-effectiveness of incorporating ECV into routine screening-related practice. 


\section{Introduction}

Screening-related colonoscopy reduces the burden of colorectal cancer (CRC) through polyp detection and removal [1]. Several quality indicators aim to standardize and optimize practice $[2,3]$. Arguably the most well-established is the adenoma detection rate (ADR), or the proportion of screening-related colonoscopies in which at least one adenoma is identified [4]. ADR is associated with the risk of post-colonoscopy CRC (PCCRC), and is therefore an important surrogate measure of the effectiveness of screening-related colonoscopy $[5,6]$.

ADR varies substantially among endoscopists [7], and patients undergoing colonoscopy by a low detector have a greater risk of PCCRC [5]. Several methods aim to improve ADR, including endoscopist feedback [8], segmental re-examination techniques [9], dynamic position changes [10], chromoendoscopy [11], and use of assistive devices. Single-use distal attachment devices fit over the colonoscope tip, and designs include caps $[12,13]$, rings $[14,15]$, and finger-like projections [16-18]. The common objective of distal attachment devices is to optimize mucosal visualization behind colonic folds, thus enhancing polyp detection.

Endocuff is a distal attachment device with finger-like projections that spread apart colonic folds to enhance ADR [19]. A newer version, Endocuff Vision (ECV), has a single row of longer arms that further optimize visualization (see Supplementary Materials). In several randomized controlled trials (RCTs), ECV has been shown to improve ADR [20-22], while potentially reducing inspection time $[20,21]$. ECV has also been shown to out-perform caps in this respect [23]. Although small pilot evaluations exist [24], no large "real-world" studies have assessed the effectiveness of ECV in non-trial settings. While only an RCT can provide robust information on efficacy, RCTs are prone to limitations in generalizability [25], and trials of technical interventions specifically may not translate into real-world clinical effectiveness [26]. Furthermore, RCTs cannot provide data on uptake, utilization, and predictors of use. Thus, we performed a carefully controlled observational study to assess the effect of ECV use on ADR in routine clinical practice, as well as to elucidate the patient-, procedure- and endoscopist-level factors associated with ECV effectiveness and uptake.

\section{Patients and methods}

\section{Study setting and design}

This observational study included data from colonoscopies performed at the Forzani \& MacPhail Colon Cancer Screening Centre (CCSC) in Calgary, Alberta, Canada between November 2018 and November 2019. The CCSC provides exclusively screening-related colonoscopies to residents in the Calgary Zone. Referrals for other indications are redirected. To be eligible at the CCSC, patients must be asymptomatic at the time of referral and fall between ages 18 and 75 . Of specific relevance to this study, patients who had major abdominal operations (e. g. segmental colonic resections for cancer) were eligible, but not within 3 months of surgery. In addition, patients with a pre- vious history of diverticulitis were eligible, but must have been off antibiotics and pain free for at least 4 weeks.

Over 40 gastroenterologists and colorectal surgeons with varying practice patterns and experience perform colonoscopies at the center. CCSC patients are allocated to endoscopists from a common queue, thereby maintaining a similar mix of cases by indication. A combination of high-definition colonoscopes (H[Q] 180/190, Olympus, Tokyo, Japan or 90i/i10, Pentax, Tokyo, Japan) were used for all cases and the choice of a regular versus smaller-caliber colonoscope was made by the endoscopist.

ECV (Olympus, Center Valley, Pennsylvania, United States) was introduced at the CCSC in May 2019 as a 6-month unitwide clinical evaluation designed by members of the CCSC's Clinical Operations Team (KC, SJH, RJH, DK). During this orientation month, all endoscopists and nursing staff received formal education on the device, and cases were performed using ECV but excluded from analysis. We examined colonoscopies performed during two time periods. Period 1 (baseline) consisted of the 6 months leading up to the implementation of ECV (November 1, 2018 to April 30, 2019). Period 2 (ECV period) spanned six months from June 1, 2019 to November 30, 2019. The decision to use ECV or to remove it during a procedure was left to the discretion of the endoscopist. However, the ECV devices (for regular or smaller caliber colonoscopes as appropriate) were pre-mounted by the endoscopy nurses unless an endoscopist requested they not be used. Our study was approved by the Health Research Ethics Board of Alberta Cancer Committee (HREBA.CC-19-0257) and was registered on clinicaltrials.gov (NCT04395352).

\section{Study patients}

All adult patients undergoing screening-related colonoscopy at the CCSC during the time periods were eligible for inclusion in the analysis. Two analysis cohorts were created: a procedural cohort and a pathology cohort, designed to calculate procedural characteristics and detection-related outcomes, respectively. Patients undergoing single balloon-assisted colonoscopy, planned advanced polypectomy including endoscopic mucosal resection (EMR) and early post-EMR surveillance were excluded. The pathology cohort, which was nested within the procedure cohort, further excluded patients under age 40. For detectionrelated outcomes in the pathology cohort we analyzed the first procedure complete to the cecum with adequate bowel preparation, defined as a Boston Bowel Preparation Score (BBPS), of 6 or higher [27].

\section{Demographic and clinical variables}

Relevant variables were acquired using CCSC's endoscopy reporting program endoPRO (Pentax Medical, Montvale, New Jersey, United States) and electronic nursing records. Patient-, procedure- and endoscopist-level variables were collected, including: patient age and sex; procedural indication (fecal immunochemical test [FIT] positive, surveillance/family history, or average risk); amounts and types of sedating medications, including use of reversal agent(s); depth of insertion, including presence or absence of cecal intubation; whether ECV was 
used; the number, estimated size, location, morphology, and histology of polyps found, biopsied or removed; procedure duration; withdrawal time (in cases without polypectomy); endoscopist specialty; and endoscopist study procedural volume.

\section{Outcome measurements}

The primary outcome was overall ADR, defined as the proportion of colonoscopies during which one or more adenomas were biopsied or removed. Histology data were abstracted from formal pathology reports by trained nurses. Secondary outcomes studied using the pathology cohort were as follows: SSADR (sessile serrated adenoma detection rate), FIT + ADR, non-FIT ADR, endoscopist-specific FIT+and non-FIT ADR and SSADR, right, transverse and left colon ADR, adenomas per colonoscopy (APC), SSAPC (sessile serrated adenomas per colonoscopy), and advanced ADR. Secondary outcomes from the procedural cohort included cecal intubation rate (CIR), withdrawal time (WT), total procedure time, nurse-assessed patient comfort scores (NAPCOMs), [28] total conscious sedation usage, and reasons for (and location of) ECV removal. Studyspecific definitions of all secondary outcomes are provided in the Supplementary Materials. Outcomes were grouped according to procedure indication (FIT +, non-FIT) and compared: 1) between standard colonoscopy and ECV-assisted colonoscopy across periods; and 2) between study periods (to assess for unit-level improvements while accounting for variable uptake and/or usage of a novel device).

\section{Statistical considerations and analyses}

Sample size was calculated based on two RCTs [20,21] demonstrating relative ADR increases of 1.13 and 1.17. Our preliminary data demonstrated a mean overall ADR of $46 \%$ in 2018 , from a mixed population including FIT + and non-FIT patients. An exact test was used to test the two-sided hypothesis that ECV is superior to unassisted colonoscopy for overall ADR. Our clinical evaluation was planned to include 6,000 patients over approximately 6 months. Assuming a two-sided alpha of 0.05 and an estimated 6,000 cases using ECV, we calculated that we would be able to demonstrate a rate ratio of 1.10 or higher (representing an increase in ADR from $46.0 \%$ to $50.6 \%$ ) with $99.9 \%$ power, a rate ratio of 1.08 or higher (representing an increase in ADR from $46.0 \%$ to $49.7 \%$ ) with $98.2 \%$ power, and a rate ratio of 1.06 or higher (representing an increase in ADR from $46.0 \%$ to $48.8 \%$ ) with $86.7 \%$ power.

All analyses were performed separately for each of the endoscopists in the study and using data for all endoscopists combined. The Mann-Whitney test was used to compare continuous outcomes by ECV use and between time periods, and Fisher's exact test was used to compare categorical outcomes. APC and SSAPC were modeled using zero-inflated negative-binomial regression. Multivariable logistic regression was used to identify independent predictors of polyp detection and to adjust for potential confounders. Crude odds ratios (ORs) of ADR and SSADR were calculated for ECV use and for each of the potential confounders, including patient age, sex, risk category (routine, increased risk, and FIT+), and study period, by univariable logistic regression. Adjusted odds ratios (AORs) of ADR and SSADR were modeled with multilevel logistic regression. All ORs and AORs were reported alongside their respective $95 \%$ confidence intervals $(\mathrm{Cl})$.

To reduce the inherent bias caused by potential confounding variables, propensity score matching in a 1:1 ratio was performed to match procedures carried out with ECV and those without. A caliper width of 0.01 without replacement was used for matching and the treatment observations with propensity scores outside the range of the propensity scores of the controls were dropped [29]. In addition, sensitivity analyses were also performed where comparisons were restricted to the ECV period and only included endoscopists performing a minimum of 10 procedures both with and without ECV.

Finally, subgroup analyses were also performed by endoscopist performance quartiles to assess their relative and absolute changes in ADR following use of ECV. These groups were defined by ranking endoscopists by their baseline ADRs among non-FIT patients. The analyses were stratified by FIT + and nonFIT risk categories, and the ADR during the ECV phase was modeled with multivariable logistic regression, using quartiles as either a continuous variable to examine trend, or a categorical variable to estimate quartile-specific OR, adjusting for patient sex and age. All statistical analyses were performed using STATA version 15.1 (StataCorp. 2017. Stata Statistical Software: Release 15. College Station, Texas, United States: StataCorp LLC) and $\mathrm{R}$ version 3.6.0 (R Foundation for Statistical Computing, Vienna, Austria).

\section{Results}

\section{Descriptive parameters}

A total of 17,562 procedures were analyzed as part of the procedural cohort. A total of 15,814 patients were analyzed as part of the pathology cohort. A flow diagram of study exclusions is provided in Fig.1. Forty-one endoscopists performed a mean of 236 standard colonoscopies and 150 ECV-assisted coIonoscopies. Men comprised $52.5 \%$ of standard colonoscopies and $53.7 \%$ of ECV-assisted colonoscopies $(p=0.16)$. Mean withdrawal time was 8.6 minutes for standard colonoscopies and 7.9 minutes for ECV-assisted colonoscopies. Detailed descriptions of patient-, endoscopist-, and procedure-related parameters, compared by ECV use across periods, are provided in - Table 1 and are compared by study period in the Supplementary Materials.

\section{Adenoma and SSA detection}

\section{Comparisons based on ECV use}

ADR was $46.7 \%$ with standard colonoscopy (baseline period, and ECV period in cases where ECV was not used) and $54.6 \%$ for colonoscopy with ECV $(P<0.001)$. This improvement was significant in both procedures for FIT + indications $(59.5 \%$ versus $66.8 \%, P<0.001)$ and non-FIT indications $(41.5 \%$ versus $49.5 \%, P<0.001)$. Detailed descriptions of detection-related outcomes, compared by ECV use across periods, are provided in $>$ Table 1 . The use of ECV was significantly associated with in- 
Total number of colonoscopies from November 2018 November 2019 (excluding May 2019) $\quad N=17,692$

- Exclusions $(\mathrm{N}=140)$

- complex ( $\geq 30 \mathrm{~mm}$ ) EMR procedures

- early EMR surveillance procedures

- single balloon colonoscopies

Total colonoscopies in procedural cohort $\mathrm{N}=17,562$

- Restricted to ages 40-75 ( $\mathrm{N}=256$ excluded)

- Restricted to first complete procedures only with BBPS $\geq 6$

( $N=1,492$ excluded $)$

Total colonoscopies/patients in pathology cohort $\mathrm{N}=15,814$
Baseline period

(Nov 12018 - Apr 30 2019) $\mathrm{N}=7,895$

FIT+

$N=2,118$

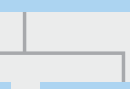

Non-FIT

$\mathrm{N}=5,777$

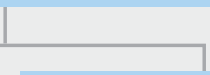

ECV period

(June 12019 - Nov 30 2019) $\mathrm{N}=7,921$

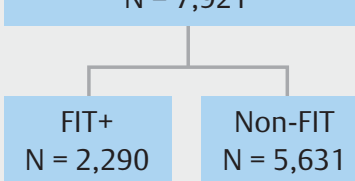

- Fig. 1 Study flow diagram showing exclusion criteria and final pathology and procedural cohorts included for primary and secondary outcomes. FIT, fecal immunochemical test; BBPS, Boston Bowel Preparation Score; EMR, endoscopic mucosal resection.

creased adjusted odds of detecting one or more adenoma(s), with an AOR of 1.24 ( $95 \% \mathrm{Cl} 1.10$ to 1.40$)$. The use of ECV was also associated with a non-significant trend toward increased odds of detecting one or more SSAs, with an AOR of 1.13 (95\% $\mathrm{Cl} 0.97$ to 1.31). ECV use was associated with higher ADR in the left colon (AOR 1.30, $95 \% \mathrm{Cl} 1.13$ to 1.48 ) and the transverse colon (AOR 1.21, $95 \% \mathrm{Cl} 1.04$ to 1.42). In addition, ECV use was associated with higher APC and SSAPC, with adjusted rate ratios of $1.20(95 \% \mathrm{Cl} 1.10,1.32)$ and $1.28(1.14,1.42)$, respectively. Although ECV use was associated with higher ADR for adenomas $<10 \mathrm{~mm}$ (AOR $1.26,95 \% \mathrm{Cl} 1.12$ to 1.41 ), it was not associated with higher ADR for adenomas $\geq 10 \mathrm{~mm}$ (AOR 1.06, $95 \% \mathrm{Cl} 0.87$ to 1.30 ). Full results of univariable and multivariable logistic regression analyses are provided in $>$ Table 2 .

\section{Comparisons between periods}

Among non-FIT patients, ADR was $41.5 \%$ in the baseline period and increased to $48.3 \%$ in the ECV period (ECV use/non-use combined, $P<0.001)$. In FIT+patients, baseline ADR was $59.5 \%$, and this increased to $65.3 \%$ in the ECV period $(P<$ $0.001)$. A significant increase was seen for SSADR in non-FIT patients, with an increase from $15.9 \%$ to $19.3 \%$ between periods $(P<0.001)$. Detailed descriptions of detection-related out- comes, compared by period and restricted to the ECV period, are provided in the Supplementary Materials.

\section{Propensity score matched analysis}

Propensity score matching greatly reduced the standardized percent bias (Supplemental Materials) and resulted in a balanced sample of 2,876 procedures (1,438 with ECV and 1,438 without ECV) for estimating the effect of ECV. The AOR of detecting one or more adenoma(s) between the ECV and nonECV matched groups was 1.16 (95\% Cl 1.00 to 1.35). Use of ECV was also associated with increased odds of detecting one or more SSA(s), with an AOR of 1.22 (95\% Cl 1.01 to 1.48). The complete results of the propensity score matched analysis is provided in the Supplementary Materials.

\section{Outcomes and usage by endoscopist characteristics}

ECV use was observed in $77.6 \%$ of cases in the ECV period. When assessing individual endoscopists' performances between periods, 28 of 32 endoscopists ( $87.5 \%$ ) meeting minimum volume thresholds, regardless of baseline performance level, observed increases in their non-FIT ADRs during the ECV period ( $\mathbf{F i g . 2}$ ). This proportion was somewhat less pronounced for FIT + ADRs, where 24 of 32 endoscopists (75.0\%) experienced improvement in the ECV period. Individual endoscopists' volumes and ADRs by patient FIT status and ECV use are provided in the Supplementary Materials. Overall, the improvements associated with ECV use were similar among low and high baseline performers in both the non-FIT and FIT + groups ( $>$ Fig.3). ECV uptake was high, with 21 of 39 endoscopists (53.8\%) adopting the device for over half their procedures from the start of the ECV period, and an additional 13 of 39 (33.3\%) adopting the device midway through the ECV period. Patterns of uptake by endoscopist characteristics are provided in the Supplementary Materials.

\section{Procedural parameters}

CIR was $98.1 \%$ in procedures with no ECV use and $98.6 \%$ in procedures with ECV use $(P=0.006)$. NAPCOM scores were 0 to 4 in $81.2 \%$ of cases with ECV use and $79.5 \%$ of cases with no ECV use, respectively $(P=0.007)$. In 312 procedures initially using ECV (4.6\%), the device was removed during the procedure. Among these cases, removal occurred most frequently in the sigmoid colon (59.0\%), followed by the rectosigmoid colon $(18.0 \%)$ and the anus (6.7\%). Patient discomfort was the most commonly cited reason for ECV removal (30.1\%). Full comparisons of procedural parameters between ECV use and non-use are provided in $>$ Table 3 . Procedures with aborted ECV use were associated with NAPCOM scores of 5 to 9 compared to 0 to 4 and with higher usage rates of fentanyl and midazolam (Supplementary Materials).

\section{Discussion}

Our study demonstrates the clinical effectiveness of Endocuff Vision without compromising procedure-related safety or efficiency in a large cohort. ECV-assisted colonoscopy was associated with significantly higher ADR and APC compared to 
Table 1 Patient and endoscopist characteristics, and procedural characteristics and outcomes by ECV use across time periods for $\mathrm{n}=15,814$ patients and $n=41$ endoscopists (pathology cohort).

\section{Patient characteristics}

Male sex (\%)

Patient age (\%)

- $40-49$

- 50-64

- $65-75$

Indications (\%)

- FIT +

- Surveillance or family history

- Average risk

\section{Endoscopist characteristics}

Specialty (\%)

- Gl

- CRS

Volume during study, mean (range)

Withdrawal time in minutes, mean (range)

Endoscopist adenoma detection rate, mean (range)

- FIT +
- Non-FIT
- Total

\section{Procedural characteristics/ outcomes}

Procedure duration (\%)

- $\leq 20$ minutes

- 21-30 minutes

- 31-60 minutes

Any sedation used (\%)

Withdrawal time ${ }^{1}$, mean (SD)

$\geq 1$ adenoma detected (\%)

- $\mathrm{FIT}+$

- Non-FIT

- Total

$\geq 1$ SSA detected (\%)

- FIT +

- Non-FIT

- Total

$\geq 1$ advanced adenoma detected (\%)

- FIT +

- Non-FIT

- Total
Standard colonoscopy $(n=9,673)$

$5,080(52.5)$
Colonoscopy with ECV

$(n=6,141)$

$3,296(53.7)$

$491(8.0)$

826 (8.5)

$6,142(63.5)$

$2,705(28.0)$

$2,595(26.8)$

$5,902(61.0)$

$1,176(12.2)$

Standard colonoscopy

$(n=41)$

32 (79.5)

9 (20.5)

$236(32-507)$

$9.3(6.3-17.3)$

$60.0(35.3-84.7)$

$41.1(17.0$ - 56.9)

$46.1(21.9-67.9)$

Standard colonoscopy

$(n=9,673)$

$6,400(68.1)$
$2,289(24.4)$
$695(7.4)$
$8,990(92.9)$
$8.6(2.7)$

$1,545(59.5)$

2,974 (41.5)

$4,519(46.7)$

406 (15.7)

$1,138(16.1)$

$1,544(16.0)$

$540(20.8)$

$337(4.8)$

877 (9.1)
3,674 (59.8)

$1,976(32.2)$

$1,813(29.5)$

$3,753(61.1)$

$<0.001$

575 (9.4)

\section{Colonoscopy with ECV}

$(n=41)$
$P$ value

0.16

$<0.001$ 
Table 1 (Continuation)

Adenomas per colonoscopy, mean (SD)

- FIT+

$1.79(3.07)$

$2.40(4.64)$

$<0.001$

- Non-FIT

$0.87(1.84)$

- Total

$1.12(2.28)$

$1.35(3.41)$

$<0.001$

$1.66(3.85)$

$<0.001$

Sessile serrated adenomas per colonoscopy, mean (SD)

\begin{tabular}{l|l}
\hline - FIT+ & $0.29(1.20)$ \\
\hline - Non-FIT & $0.26(0.83)$ \\
\hline - Total & $0.27(0.94)$
\end{tabular}

$0.32(1.11)$

0.39

$0.41(1.37)$

$<0.001$

$0.38(1.30)$

$<0.001$

FIT, fecal immunochemical test; ECV, Endocuff Vision; GI, gastroenterology; CRS, colorectal surgery;

SSA, sessile serrated adenoma; advanced adenoma is one measuring $10 \mathrm{~mm}$ or greater, or with tubulovillous/villous pathology or high-grade dysplasia irrespective of size.

${ }^{1}$ Calculated from procedures with no pathology.

- Table 2 Crude and adjusted odds ratios for adenoma and sessile serrated adenoma detection by selected variables.

\begin{tabular}{|c|c|c|c|c|}
\hline Crude odds ratios (OR) & Adenoma & $95 \% \mathrm{Cl}$ & SSA & $95 \% \mathrm{Cl}$ \\
\hline Male sex & 1.90 & $1.79,2.03$ & 0.96 & $0.88,1.04$ \\
\hline Age & 1.15 & $1.09,1.21$ & 1.02 & $0.96,1.10$ \\
\hline Age $^{2}$ (quadratic term) & 0.9992 & $0.9987,0.9996$ & 0.9999 & $0.9993,1.0004$ \\
\hline \multicolumn{5}{|l|}{ Risk category } \\
\hline - Average risk (reference) & 1.00 & $N / A$ & 1.00 & $N / A$ \\
\hline - Surveillance or family history & 1.23 & $1.11,1.37$ & 1.26 & $1.10,1.45$ \\
\hline . FIT+ & 2.45 & $2.19,2.75$ & 1.09 & $0.93,1.27$ \\
\hline Study period (ECV vs. baseline) & 1.32 & $1.24,1.41$ & 1.20 & $1.10,1.30$ \\
\hline ECV use (across periods) & 1.37 & $1.29,1.48$ & 1.23 & $1.13,1.33$ \\
\hline Adjusted odds ratio (AOR) & Adenoma & $95 \% \mathrm{Cl}$ & SSA & $95 \% \mathrm{Cl}$ \\
\hline ECV use (across periods) & 1.24 & $1.10,1.40$ & 1.13 & $0.97,1.31$ \\
\hline \multicolumn{5}{|l|}{ ECV use, by colonic segment } \\
\hline - Left colon & 1.30 & $1.13,1.48$ & 1.37 & $0.99,1.88$ \\
\hline - Transverse colon & 1.21 & $1.04,1.42$ & 1.13 & $0.88,1.45$ \\
\hline - Right colon & 1.01 & $0.89,1.15$ & 1.15 & $0.96,1.38$ \\
\hline \multicolumn{5}{|l|}{ ECV use, by adenoma size } \\
\hline - Small adenoma $(<10 \mathrm{~mm})$ & 1.26 & $1.12,1.41$ & - & - \\
\hline - Large adenoma ( $\geq 10 \mathrm{~mm}$ ) & 1.06 & $0.87,1.30$ & - & - \\
\hline Adjusted rate ratio (ARR) & APC & $95 \% \mathrm{Cl}$ & SSAPC & $95 \% \mathrm{Cl}$ \\
\hline ECV use (across periods) & 1.20 & $1.10,1.32$ & 1.28 & $1.14,1.42$ \\
\hline
\end{tabular}

$\mathrm{CI}$, confidence intervals; SSA, sessile serrated adenoma; FIT, fecal immunochemical test; ECV, Endocuff Vision; left colon, all segments from splenic flexure distally; right colon, all segments from hepatic flexure proximally; APC, adenomas per colonoscopy; SSAPC, sessile serrated adenomas per colonoscopy.

standard colonoscopy. Furthermore, all relevant procedural parameters remained unhindered by ECV use, including CIR, mean withdrawal time, overall procedure duration, sedation use, and patient comfort.

A primary goal of screening-related colonoscopy is to detect and remove premalignant adenomas [1]; therefore, efforts to measure and improve ADR are important. ADR is affected by patient-related factors, but also varies widely between endoscopists [7]. Thus, the ideal intervention for ADR should be broadly applicable to as many patients and endoscopists as possible. Interventions targeting endoscopists are effective but require planning and are costly $[8,30]$. Distal attachment 

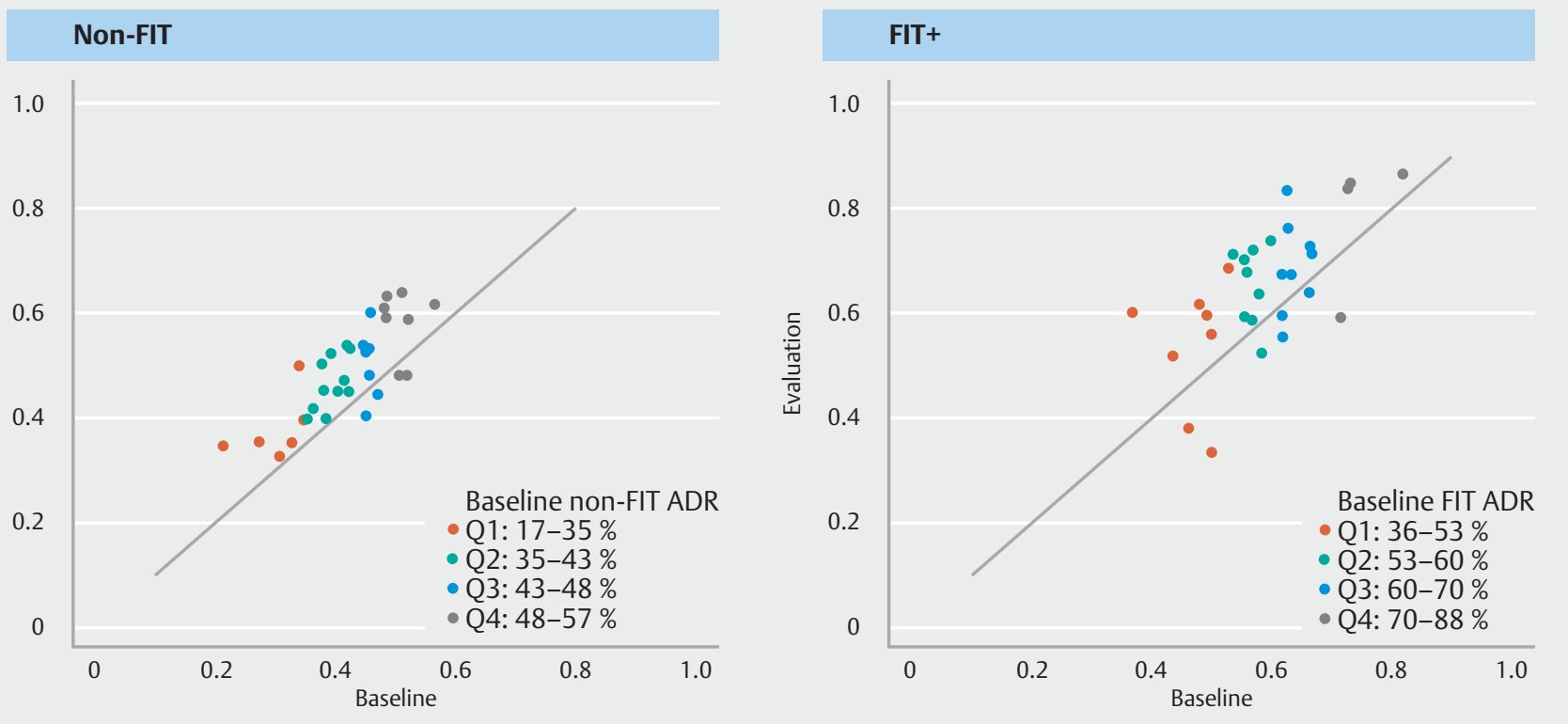

Fig. 2 Scatterplots of endoscopist ADRs by study period, stratified by patient FIT status and colored by physician baseline ADR quartiles among non-FIT patients. Endoscopists with fewer than 20 procedures in any of the four subcategories $(n=10)$ were removed.
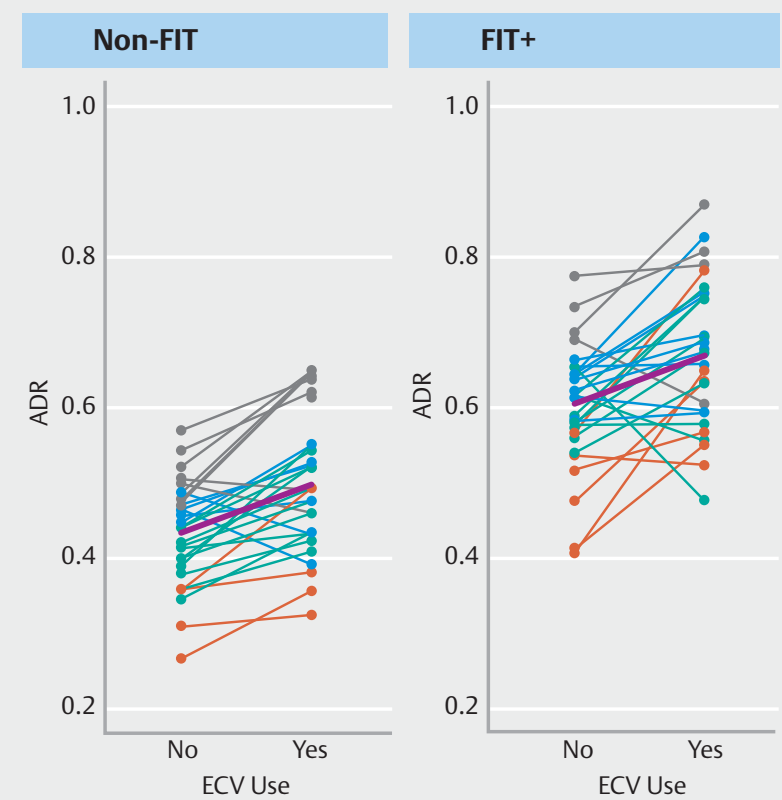

Baseline non-FIT ADR

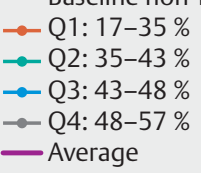

$\rightarrow$ Q1: $17-35 \%$

$\rightarrow$ Q3: $43-48 \%$

- Average

Fig. 3 Line graphs of endoscopist ADRs by use of ECV, stratified by patient FIT status and colored by physician baseline non-FIT ADR quartiles. Endoscopists with fewer than 20 procedures in any of the four subcategories $(n=13)$ were removed. devices are simple to use [12-14], and ECV in particular is efficacious in improving ADR [20-23]. No large "real-world" studies have assessed the effectiveness of ECV. We demonstrated a significant improvement in ADR with ECV use (54.6\%) compared to non-use $(46.7 \%)$, consistent with that demonstrated in RCTs [20-22]. Furthermore, our ECV removal rate of $4.6 \%$ is consistent with a previously reported range of $0.6 \%$ to $4.1 \%$ $[20,22]$. However, given that ECV use was discretionary and ultimately used in $78 \%$ of cases, this rate may have underestimated the removal rate if ECV use was mandated. CIR, while high at $98.1 \%$ with standard colonoscopy, was similar with ECV use (98.6\%); however, CIR may have been lower with ECV if its use had been mandated in all cases. Furthermore, mean endoscopist withdrawal time went from 9.2 minutes in the baseline period to 8.8 minutes in the ECV period, indicating that improvements in detection were not observed as a result of longer examination times.

In a recent $R C T$, high performers derived the greatest benefit from ECV use [22]. In our study, no significant trends were observed regarding preferential improvements by baseline performance levels. ECV uptake was high, with no incentives for use or consequences for lack of use. Even with non-universal uptake, we observed a significant improvement in overall ADR from the baseline period (46.3\%) to the ECV period $(53.2 \%)$. Therefore, we demonstrate that ECV, when introduced at an institutional level, is broadly effective and practical. Furthermore, our results are generalizable given that over 40 endoscopists and 15,000 patients were included.

The cost of devices is also important when considering clinical implementation. Though associated with minor incremental increases in the cost of a screening colonoscopy [31], ECV devices are nevertheless costly when applied across a large program. However, given that each $1 \%$ increase in ADR has been 
D Table 3 Procedural parameters compared across periods for 17,557 procedures (procedural cohort). ${ }^{1}$

\begin{tabular}{|c|c|c|c|}
\hline Variables, $\mathrm{N}(\%)$ or mean (SD) & $\begin{array}{l}\text { Standard colonoscopy } \\
(n=10,763)\end{array}$ & $\begin{array}{l}\text { Colonoscopy with ECV } \\
(n=6,794)\end{array}$ & $P$ value \\
\hline Cecum intubated (\%) & $10,555(98.1)$ & $6,700(98.6)$ & 0.006 \\
\hline \multicolumn{4}{|l|}{ NAPCOMs } \\
\hline - $0-4(\%)$ & $8,356(79.5)$ & $5,407(81.2)$ & 0.007 \\
\hline - 5-9(\%) & $2,155(20.5)$ & $1,254(18.8)$ & \\
\hline - Mean (SD) & $2.7(2.1)$ & $2.6(2.0)$ & $<0.001$ \\
\hline \multicolumn{4}{|l|}{ Sedation used } \\
\hline - Fentanyl in mcg, mean (SD) & $55.6(24.6)$ & $54.5(24.1)$ & 0.004 \\
\hline - Midazolam in mg, mean (SD) & $3.1(1.2)$ & $3.1(1.2)$ & $<0.001$ \\
\hline Procedures with aborted ECV use & $\mathrm{N} / \mathrm{A}$ & $312(4.6)$ & $N / A$ \\
\hline \multicolumn{3}{|l|}{ Location of aborted ECV use (\%) } & \multirow[t]{9}{*}{$N / A$} \\
\hline - Anus & & $21(6.7)$ & \\
\hline - Rectosigmoid & & $56(18.0)$ & \\
\hline - Sigmoid & & $184(59.0)$ & \\
\hline - Descending colon & & $19(6.1)$ & \\
\hline - Splenic flexure & & $9(2.9)$ & \\
\hline - Transverse colon & & $13(4.1)$ & \\
\hline - Hepatic flexure & & $2(0.6)$ & \\
\hline - Ascending colon & & $8(2.5)$ & \\
\hline \multicolumn{3}{|c|}{ Endoscopist rationale for aborted ECV use } & \multirow[t]{8}{*}{$N / A$} \\
\hline - Colonic narrowing & & $34(10.9)$ & \\
\hline - Diverticulosis & & $40(12.8)$ & \\
\hline - Patient discomfort & & $94(30.1)$ & \\
\hline - Poor bowel preparation & & $35(11.2)$ & \\
\hline - Unable to intubate anus & & $58(18.6)$ & \\
\hline - Colonoscope changed & & $10(3.2)$ & \\
\hline - Other & & $41(13.1)$ & \\
\hline
\end{tabular}

associated with a $3 \%$ decrease in CRC risk [5] there is potential for long-term cost savings to the system with ECV use. Indeed, this has been shown in a cost-effectiveness analysis of the older generation of Endocuff [31], whose incremental ADR improvement was less impressive than the newer-generation ECV. Conversely, our study suggests that ECV use increases ADR for adenomas $<10 \mathrm{~mm}$, but not for adenomas $\geq 10 \mathrm{~mm}$, the latter of which carry greater malignant potential [32]. Future economic analyses need to account for these important factors.

The increase in ADR that we observed is greater than that reported in the clinical trial literature. This could be partly due to the observational design of our study, to which temporal trends in ADR and confounding may have contributed. However, the magnitude of effect observed despite the short 1-month time interval between baseline and ECV periods makes it unlikely that the increase in ADR with ECV use resulted from unrelated temporal increases in ADR over time. We also adjusted for study period in our multivariable models, demonstrating that ECV use was independently associated with improvements in ADR. Furthermore, our results were unchanged when analyzed within a subgroup analysis where ECV use and non-use was restricted to the ECV period. Endoscopists at our center receive report cards biannually which include their ADR, and thus, they are continually being observed. In addition, our findings occurred despite no increase in mean withdrawal time. Thus, it is unlikely that the Hawthorne effect influenced our results. 
Although nearly $80 \%$ of procedures were completed using ECV, endoscopists were permitted to choose when to use the device, and thus, confounding could still have been introduced. However, we controlled for important potential confounders, including age, sex, procedure indication and study period, but did not have data on other potential confounders such as body mass index (BMI). Elevated BMI increases the risk of colorectal adenomas [33], while colonoscopy in patients with either very high or low BMI can be more difficult [34], which could have influenced decisions to use ECV. To further control for confounding, we also conducted a propensity score-matched analysis intended to mimic randomization by generating a balanced distribution of covariates among a group of treated (ECV) and control (no ECV) patients [35]. As this methodology required us to use only the ECV period data our sample size was drastically reduced. Nevertheless, while the effect size of ECV on ADR dropped, it remained substantial and statistically significant. Regardless, even if highly likely that our observed improvements in ADR are primarily due to ECV, we were ultimately unable to control for all known and unknown confounders due to our nonclinical trial design.

Our study has other potential limitations. We could not evaluate the performance of ECV in detecting pathology in procedures when bowel cleansing was poor, given that such patients were excluded from our pathology cohort. It is routine practice at our center to reschedule patients with inadequate bowel prep, given the risk of missing significant pathology. However, patients with poor bowel preparation were included in our procedural cohort. It may be of future interest to assess ECV performance in this clinical context, whereby ECV could improve visualization or aid in the completion of difficult colonoscopies resulting from colonic redundancy. This was also a single-center study, but it included over 40 different endoscopists (both gastroenterologists and surgeons) of varying experience and practice focus (academics and non-academics) on over 15,000 patients with a variety of screening-related indications.

\section{Conclusions}

In conclusion, this large real-world study demonstrated that ECV use is associated with significant improvements in adenoma and SSA detection and is not associated with any measurable negative impacts on patient safety, procedural completeness, or procedural efficiency. ECV use appeared to be beneficial in the majority of studied major endoscopist- and patientrelated categories. Further research is required to clearly elucidate the cost-effectiveness of ECV use to guide optimal healthcare resource utilization.

\section{Acknowledgements}

This study was partially funded by the Calgary Health Trust. No funding or study input was received from the device manufacturer.
Competing interests

The authors declare that they have no conflict of interest.

\section{References}

[1] Zauber AG, Winawer S], O'Brien M] et al. Colonoscopic polypectomy and long-term prevention of colorectal-cancer deaths. N Engl J Med 2012; 366: 687-696

[2] Rees C], Thomas Gibson S, Rutter MD et al. UK key performance indicators and quality assurance standards for colonoscopy. Gut 2016; 65: 1923-1929

[3] Rex DK, Schoenfeld PS, Cohen J et al. Quality indicators for colonoscopy. Am J Gastroenterol 2015; 110: 72-90

[4] Rex DK, Bond JH, Winawer S et al. Quality in the technical performance of colonoscopy and the continuous quality improvement process for colonoscopy: recommendations of the U.S. Multi-Society Task Force on Colorectal Cancer. Am J Gastroenterol 2002; 97: 12961308

[5] Corley DA, Jensen CD, Marks AR et al. Adenoma detection rate and risk of colorectal cancer and death. N Engl J Med 2014; 370: 12981306

[6] Kaminski MF, Regula J, Kraszewska E et al. Quality indicators for colonoscopy and the risk of interval cancer. N Engl J Med 2010; 362: 1795-1803

[7] Hilsden RJ, Rose SM, Dube C et al. Defining and applying locally relevant benchmarks for the adenoma detection rate. Am J Gastroenterol 2019; 114: 1315-1321

[8] Bishay K, Causada-Calo N, Scaffidi MA et al. Endoscopist feedback is associated with improvements in colonoscopy quality indicators: a systematic review and meta-analysis. Gastrointest Endosc 2020; 92: $1030-1040$

[9] Desai M, Bilal M, Hamade $\mathrm{N}$ et al. Increasing adenoma detection rates in the right side of the colon comparing retroflexion with a second forward view: a systematic review. Gastrointest Endosc 2019; 89: 453-459.e453

[10] Lee SW, Chang JH, Ji JS et al. Effect of dynamic position changes on adenoma detection during colonoscope withdrawal: a randomized controlled multicenter trial. Am J Gastroenterol 2016; 111: 63-69

[11] Desai M, Viswanathan L, Gupta $\mathrm{N}$ et al. Impact of electronic chromoendoscopy on adenoma miss rates during colonoscopy: a systematic review and meta-analysis. Dis Colon Rectum 2019; 62: 1124-1134

[12] Pohl H, Bensen SP, Toor A et al. Cap-assisted colonoscopy and detection of Adenomatous Polyps (CAP) study: a randomized trial. Endoscopy 2015; 47: 891-897

[13] Othman MO, Zhang D, Elhanafi S et al. Cap-assisted colonoscopy increases detection of advanced adenomas and polyps. Am J Med Sci 2017; 353: 367-373

[14] Rex DK, Repici A, Gross SA et al. High-definition colonoscopy versus Endocuff versus EndoRings versus full-spectrum endoscopy for adenoma detection at colonoscopy: a multicenter randomized trial. Gastrointest Endosc 2018; 88: 335-344.e332

[15] Hassan C, Senore C, Manes G et al. Diagnostic yield and miss rate of EndoRings in an organized colorectal cancer screening program: the SMART (Study Methodology for ADR-Related Technology) trial. Gastrointest Endosc 2019; 89: 583-590.e581

[16] Bhattacharyya R, Chedgy F, Kandiah K et al. Endocuff-assisted vs. standard colonoscopy in the fecal occult blood test-based UK Bowel Cancer Screening Programme (E-cap study): a randomized trial. Endoscopy 2017; 49: 1043-1050 
[17] Baek MD, Jackson CS, Lunn J et al. Endocuff assisted colonoscopy significantly increases sessile serrated adenoma detection in veterans. J Gastrointest Oncol 2017; 8: 636-642

[18] Birk JW, Anderson JC. Endocuff and detection of colorectal adenomas: results of a randomized controlled study. Gastroenterology 2016; 150: 1684-1686

[19] Chin M, Karnes W, Jamal MM et al. Use of the Endocuff during routine colonoscopy examination improves adenoma detection: A meta-analysis. World J Gastroenterol 2016; 22: 9642-9649

[20] Ngu W, Bevan R, Tsiamoulos Z et al. Improved adenoma detection with Endocuff Vision: the ADENOMA ransomised controlled trial. Gut 2018; 66: 1-9

[21] Rex DK, Slaven JE, Garcia J et al. Endocuff vision reduces inspection time without decreasing lesion detection: a clinical randomized trial. Clin Gastroenterol Hepatol 2020; 18: 158-162.e151

[22] Karsenti D, Tharsis G, Perrot B et al. Adenoma detection by Endocuffassisted versus standard colonoscopy in routine practice: a clusterrandomised crossover trial. Gut 2020; 69: 2159-2164

[23] Rameshshanker R, Tsiamoulos Z, Wilson A et al. Endoscopic cuff-assisted colonoscopy versus cap-assisted colonoscopy in adenoma detection: randomized tandem study-DEtection in Tandem Endocuff Cap Trial (DETECT). Gastrointest Endosc 2020; 91: 894-904.e891

[24] Tsiamoulos ZP, Misra R, Rameshshanker R et al. Impact of a new distal attachment on colonoscopy performance in an academic screening center. Gastrointest Endosc 2018; 87: 280-287

[25] Sherman RE, Anderson SA, Dal Pan G] et al. Real-world evidence what is it and what can it tell us? New Engl J Med 2016; 375: 22932297

[26] Forbes N, Hilsden RJ, Lethebe BC et al. Prophylactic endoscopic clipping does not prevent delayed postpolypectomy bleeding in routine clinical practice: a propensity score-matched cohort study. Am J Gastroenterol 2020; 115: 774-782

[27] Calderwood AH, Jacobson BC. Comprehensive validation of the Boston Bowel Preparation Scale. Gastrointest Endosc 2010; 72: 686-692

[28] Rostom A, Ross ED, Dube C et al. Development and validation of a nurse-assessed patient comfort score for colonoscopy. Gastrointest Endosc 2013; 77: 255-261

[29] Austin PC. Some methods of propensity-score matching had superior performance to others: results of an empirical investigation and Monte Carlo simulations. Biom J 2009; 51: 171-184

[30] Causada-Calo N, Gonzalez-Moreno G, Bishay K et al. Educational interventions are associated with improvements in colonoscopy quality indicators: a systematic review and meta-analysis. Endosc Int Open 2020; 8: E1321-E1331

[31] Yu TM, Tradonsky A, Tang J et al. Cost-effectiveness of adding Endocuff(®) to standard colonoscopies for interval colorectal cancer screening. Clinicoecon Outcomes Res 2019; 11: 487-504

[32] Muto T, Bussey HJ, Morson BC. The evolution of cancer of the colon and rectum. Cancer 1975; 36: 2251-2270

[33] Ben Q, An W, jiang Y et al. Body mass index increases risk for colorectal adenomas based on meta-analysis. Gastroenterology 2012; 142: 762-772

[34] Moon SY, Kim BC, Sohn DK et al. Predictors for difficult cecal insertion in colonoscopy: The impact of obesity indices. World J Gastroenterol 2017; 23: 2346-2354

[35] Austin PC. An introduction to propensity score methods for reducing the effects of confounding in observational studies. Multivar Behav Res 2011; 46: 399-424 